\title{
Height-Averaged Navier-Stokes Solver for Hydrodynamic Lubrication
}

\author{
Hannes Holey ${ }^{1,2}\left(\right.$ A $\cdot$ Andrea Codrignani ${ }^{2,3} \cdot$ Peter Gumbsch ${ }^{1,3} \cdot$ Lars Pastewka $^{2,4}$
}

Received: 29 September 2021 / Accepted: 8 February 2022 / Published online: 5 March 2022

(c) The Author(s) 2022

\begin{abstract}
The cornerstone of thin-film flow modeling is the Reynolds equation-a lower-dimensional representation of the NavierStokes equation. The derivation of the Reynolds equation is based on explicit assumptions about the constitutive behavior of the fluid that prohibit applications in multiscale scenarios based on measured or atomistically simulated data. Here, we present a method that treats the macroscopic flow evolution and the calculation of local cross-film stresses as separate yet coupled problems - the so-called macro and micro problem. The macro problem considers mass and momentum balance for compressible fluids in a height-averaged sense and is solved using a time-explicit finite-volume scheme. Analytical solutions for the micro problem are derived for common constitutive laws and implemented into the Height-averaged Navier-Stokes (HANS) solver. We demonstrate the validity of our solver on examples, including mass-conserving cavitation, inertial effects, wall slip, and non-Newtonian fluids. The presented method is not limited to these fixed-form relations and may therefore be useful for testing constitutive relations obtained from experiment or simulation.
\end{abstract}

Keywords Fluid mechanics methods $\cdot$ Hydrodynamic lubrication $\cdot$ Cavitation in hydrodynamics $\cdot$ Compressibility in hydrodynamics

\section{Introduction}

Although the fundamental equation for hydrodynamic lubrication was derived by Reynolds [1] almost 140 years ago, accurate descriptions of thin-film fluid flow are still a research topic of ongoing interest. Major challenges in modeling arise in elastohydrodynamic lubrication (EHL), where a plethora of effects, such as non-Newtonian fluid behavior,

Hannes Holey

hannes.holey@kit.edu

Lars Pastewka

lars.pastewka@imtek.uni-freiburg.de

1 Institute for Applied Materials, Karlsruhe Institute of Technology, Straße am Forum 7, 76131 Karlsruhe, Germany

2 Department of Microsystems Engineering (IMTEK), University of Freiburg, Georges-Koehler-Allee 103, 79110 Freiburg, Germany

3 Fraunhofer IWM, Wöhlerstraße 11, 79108 Freiburg, Germany

4 Cluster of Excellence livMatS, Freiburg Center for Interactive Materials and Bioinspired Technologies, University of Freiburg, Georges-Koehler-Allee 105, 79110 Freiburg, Germany surface roughness, wall slip, or cavitation, has to be considered. An extensive overview of the field of EHL is given in the review articles by Lugt and Morales-Espejel [2] and Gropper et al. [3], the latter focusing on textured surfaces.

The derivation of the Reynolds equation is based on an asymptotic analysis of the incompressible Navier-Stokes equation under the assumption that the gap height is small compared to the lateral dimensions. As a result, inertial and body force terms can be neglected and the fluid pressure does not depend on the gap height coordinate. This allows integration of the momentum equations in the gap height coordinate to obtain the flow velocity profiles. Integration of the mass balance using these velocity profiles leads to the Reynolds equation for incompressible and isoviscous fluids [4].

However, fluid compressibility and pressure-dependent viscosity cannot be ignored under severe loading conditions, such as in EHL contacts. It is therefore common practice to introduce constitutive relations for the pressure-dependent density and viscosity to the asymptotic analysis a posteriori. Yet, this can lead to wrong predictions for piezoviscous fluids, where the viscosity strongly depends on pressure [5-7].

Recently, Almqvist et al. [8] presented a compressible Reynolds equation for pressure-density relations that take 
the form of a power law. This includes the isothermal ideal gas law, the constant bulk modulus equations of state, as well as the Dowson-Higginson equation [9] for mineral base oils. They found that for weakly compressible fluids, lowerdimensional formulations of the Navier-Stokes equations can be derived and that inertia and body force terms may be neglected based on the thin-film assumption. However, for the ideal gas, inertia and body force terms do not automatically vanish, which is why the additional assumptions that these terms are negligibly small have to be made.

In this paper, we present a novel method that requires no a priori assumptions on the form of the constitutive equations. Our splitting approach for the conserved variables in the three-dimensional mass and momentum balance leads to two separate problems: a macro problem, which describes the time evolution of height-averaged variables in two spatial dimensions, and a micro problem, which determines the local stress state given the macroscopic flow and boundary conditions. The solution procedure for the macro problem is therefore independent of the type of constitutive equation used in the micro problem.

We demonstrate the validity of our method by solving the micro problem for Newtonian and generalized Newtonian fluids and compare the results from our transient finite-volume implementation with various Reynolds-based solutions from the literature. Moreover, the formulation in terms of conserved variables allows a flexible implementation of cavitation models through the equation of state. We also demonstrate Navier slip boundary conditions for the investigation of lubrication with heterogeneous surface wettability.

\section{Governing Equations}

We start with the local form of mass and momentum balance, which is given by the partial differential equation

$\partial_{t} \boldsymbol{q}=-\operatorname{div} \mathbb{F}(\boldsymbol{q})$,

where $\boldsymbol{q}$ denotes the 4-vector of densities of conserved variables (mass and momentum) and $\mathbb{F}(\boldsymbol{q})$ denotes the corresponding flux functions, represented by a $4 \times 3$ matrix

$\boldsymbol{q}=\left(\begin{array}{l}\rho \\ \vec{j}\end{array}\right), \quad \mathbb{F}(\boldsymbol{q})=\left(\begin{array}{c}\vec{j} \\ \frac{1}{\rho}(\vec{j} \otimes \vec{j})+p \underline{1}-\underline{\tau}(\boldsymbol{q})\end{array}\right)$,

where $\rho$ is the density. Note that the divergence operator in Eq. (1) acts on the column index $[\operatorname{div} \mathbb{A}]_{i}=\partial_{j} A_{i j}$. The first row of $\mathbb{F}$ contains the components of the mass flux vector $\vec{j}$, representing mass transport. Momentum transport is encoded in the convective acceleration term $(\vec{j} \otimes \vec{j}) / \rho$, where $\otimes$ defines the outer product between two vectors as $[\vec{a} \otimes \vec{b}]_{i j}=a_{i} b_{j}$, and the pressure term $p \underline{1}$, where 1 denotes the $3 \times 3$ unit matrix. Note that here and in the following we use arrows (e.g., $\vec{j}$ ) for tensors of rank 1 (represented as Cartesian 3-vectors), underlines (e.g., $\underline{\tau}$ ) for tensors of rank 2 (represented as $3 \times 3$ matrices), bold font (e.g., q) for 4-vectors, and open fonts (e.g., $\mathbb{F}$ ) for $4 \times 3$ matrices.

In a compressible fluid, pressure is defined through the equation of state (EOS). Here, we consider only isothermal conditions such that the energy equation can be omitted and the EOS is given by the pressure-density relation $p=p(\rho)$. Finally, the viscous stress tensor $\tau$ is a function of the conserved variables, which shall not be further specified at this point.

Let us now assume that a general solution to Eq. (1) can be split into a time-dependent macro solution $\overline{\mathbf{q}}$, which is obtained by averaging the balance equations over the gap height (see Sect. 2.1), and a stationary micro solution $\delta \mathbf{q}$ that resolves the conserved variables in the gap height coordinate locally (see Sect. 2.3). We can then formally write

$\mathbf{q}(x, y, z, t)=\overline{\mathbf{q}}(x, y, t)+\delta \mathbf{q}(z)$.

The implication of this splitting will become evident in the solution of the micro problem.

In the following, we treat the solution of the macro and micro problem separately - a situation which is comparable to multiscale simulations, where a more accurate method is used to obtain a "micro solution" which governs the coarser method in an either sequential or concurrent manner.

\subsection{Macro Problem}

To arrive at a two-dimensional description we take an average of Eq. (1) in z-direction

$\frac{1}{h} \int_{h_{1}}^{h_{2}} \partial_{t} \boldsymbol{q} \mathrm{d} z=-\frac{1}{h} \int_{h_{1}}^{h_{2}}\left(\partial_{x} \boldsymbol{f}_{x}+\partial_{y} f_{y}+\partial_{z} f_{z}\right) \mathrm{d} z$,

where $\boldsymbol{f}_{i}$ denotes the $i$-th column of the flux function matrix $\mathbb{F}$ (i.e., the flux in direction $i), h_{1}\left(x_{1}, y_{1}\right)$ and $h_{2}\left(x_{2}, y_{2}\right)$ are the height profiles of the lower and upper surface, respectively, and their difference is denoted with $h$ as shown in Fig. 1a. Note that these profiles are expressed in coordinate systems that are attached to the walls, which move relative to the global reference frame with constant velocities $\mathbf{U}_{1}=\left(U_{1}, V_{1}, 0\right)^{\top}$ and $\mathbf{U}_{2}=\left(U_{2}, V_{2}, 0\right)^{\top}$.

Applying the Leibniz integral rule for differentiation under the integral sign and the product rule, the 1.h.s. of Eq. (4) reads

$$
\begin{aligned}
\int_{h_{1}}^{h_{2}} \partial_{t} \boldsymbol{q} \mathrm{d} z & =\partial_{t} \int_{h_{1}}^{h_{2}} \boldsymbol{q} \mathrm{d} z-\left.\boldsymbol{q}\right|_{z=h_{2}} \frac{\mathrm{d} h_{2}}{\mathrm{~d} t}+\left.\boldsymbol{q}\right|_{z=h_{1}} \frac{\mathrm{d} h_{1}}{\mathrm{~d} t} \\
& =h \partial_{t} \overline{\boldsymbol{q}}+\left(\overline{\boldsymbol{q}}-\left.\boldsymbol{q}\right|_{z=h_{2}}\right) \frac{\mathrm{d} h_{2}}{\mathrm{~d} t}-\left(\overline{\boldsymbol{q}}-\left.\boldsymbol{q}\right|_{z=h_{1}}\right) \frac{\mathrm{d} h_{1}}{\mathrm{~d} t},
\end{aligned}
$$

and similarly, for the first term on the r.h.s of Eq. (4), we obtain 
(a)

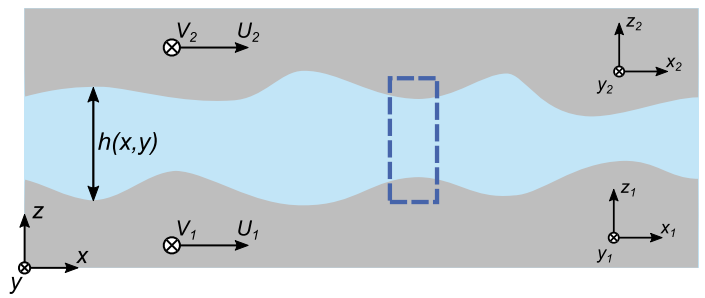

(b)

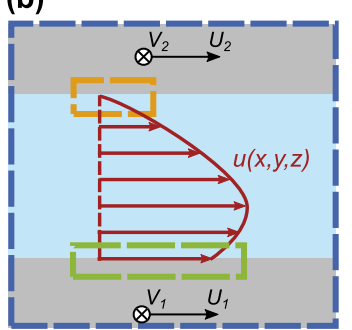

(c)

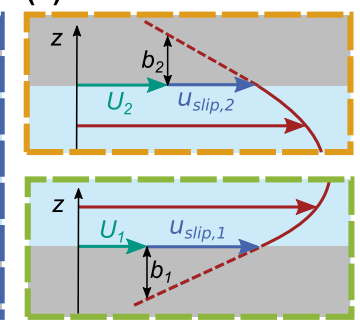

Fig. 1 Sketch of the lubrication gap with height profiles of the lower and upper surface given in the local coordinate systems $\left(x_{i}, y_{i}, z_{i}\right)$, moving with constant velocities $\mathbf{U}_{\mathbf{i}}=\left(U_{i}, V_{i}, 0\right)^{\top}$ and $i \in[1,2]$. Panel a represents the macro problem and the micro problem is shown as a blue box in (a) or magnified in panel (b). The solution of the micro problem does not depend on the surface height gradients anymore. Deviation from no-slip boundary conditions is considered through the Navier slip length on the upper and lower surface respectively (c)

$$
\begin{aligned}
\int_{h_{1}}^{h_{2}} \partial_{x} \boldsymbol{f}_{x} \mathrm{~d} z & =\partial_{x} \int_{h_{1}}^{h_{2}} \boldsymbol{f}_{x} \mathrm{~d} z-\left.\boldsymbol{f}_{x}\right|_{z=h_{2}} \frac{\partial h_{2}}{\partial x}+\left.\boldsymbol{f}_{x}\right|_{z=h_{1}} \frac{\partial h_{1}}{\partial x} \\
& =h \partial_{x} \overline{\boldsymbol{f}}_{x}+\left(\overline{\boldsymbol{f}}_{x}-\left.\boldsymbol{f}_{x}\right|_{z=h_{2}}\right) \frac{\partial h_{2}}{\partial x}-\left(\overline{\boldsymbol{f}}_{x}-\left.\boldsymbol{f}_{x}\right|_{z=h_{1}}\right) \frac{\partial h_{1}}{\partial x},
\end{aligned}
$$

where overbars denote height averages $\bar{\phi}=\frac{1}{h} \int_{h_{1}}^{h_{2}} \phi \mathrm{d} z$ (see Appendix A for more details). The second term of Eq. (4) is identical but has $x$ replaced by $y$. The third term under the integral on the r.h.s. of Eq. (4) can be evaluated directly at the fluid-wall boundaries

$\frac{1}{h} \int_{h_{1}}^{h_{2}} \partial_{z} f_{z} \mathrm{~d} z=\frac{1}{h}\left(\left.\boldsymbol{f}_{z}\right|_{z=h_{2}}-\left.\boldsymbol{f}_{z}\right|_{z=h_{1}}\right)$.

Hence, the averaged scheme is composed of a two-dimensional divergence operator acting on height-averaged flux functions and of terms containing both averaged and unaveraged flux functions, the latter evaluated at the top and bottom walls. The terms outside of the divergence operator can be regarded as geometrical source terms due to the reduction of dimensionality.

The averaged scheme reads

$\partial_{t} \overline{\boldsymbol{q}}=-\partial_{x} \bar{f}_{x}-\partial_{y} \bar{f}_{y}-\boldsymbol{s}$,

$$
\begin{aligned}
\boldsymbol{s} & =\frac{1}{h}\left[\frac{\partial h_{2}}{\partial x}\left(\overline{\boldsymbol{f}}_{x}-\left.\boldsymbol{f}_{x}\right|_{z=h_{2}}\right)-\frac{\partial h_{1}}{\partial x}\left(\overline{\boldsymbol{f}}_{x}-\left.\boldsymbol{f}_{x}\right|_{z=h_{1}}\right)\right. \\
& +\frac{\partial h_{2}}{\partial y}\left(\overline{\boldsymbol{f}}_{y}-\left.\boldsymbol{f}_{y}\right|_{z=h_{2}}\right)-\frac{\partial h_{1}}{\partial y}\left(\overline{\boldsymbol{f}}_{y}-\left.\boldsymbol{f}_{y}\right|_{z=h_{1}}\right) \\
& \left.-\frac{\mathrm{d} h_{2}}{\mathrm{~d} t}\left(\overline{\boldsymbol{q}}-\left.\boldsymbol{q}\right|_{z=h_{2}}\right)+\frac{\mathrm{d} h_{1}}{\mathrm{~d} t}\left(\overline{\boldsymbol{q}}-\left.\boldsymbol{q}\right|_{z=h_{1}}\right)+\left.\boldsymbol{f}_{z}\right|_{z=h_{2}}-\left.\boldsymbol{f}_{z}\right|_{z=h_{1}}\right] .
\end{aligned}
$$

The total time derivative for the upper $(i=2)$ and lower $(i=1)$ rigid surfaces can be written as

$\frac{\mathrm{d} h_{i}}{\mathrm{~d} t}=\frac{\partial h_{i}}{\partial x} U_{i}+\frac{\partial h_{i}}{\partial y} V_{i}$.

Without loss of generality, we assume that the lower wall is flat $\left(h_{1}=\right.$ const. $)$ and the upper wall is stationary $\left(\mathbf{U}_{2}=\mathbf{0}\right)$. Then, the expression for the source term simplifies significantly to

$\boldsymbol{s}=\frac{1}{h}\left[\frac{\partial h}{\partial x}\left(\overline{\boldsymbol{f}}_{x}-\left.\boldsymbol{f}_{x}\right|_{z=h_{2}}\right)+\frac{\partial h}{\partial y}\left(\overline{\boldsymbol{f}}_{y}-\left.\boldsymbol{f}_{y}\right|_{z=h_{2}}\right)+\left.\boldsymbol{f}_{z}\right|_{z=h_{2}}-\left.\boldsymbol{f}_{z}\right|_{z=h_{1}}\right]$.

We use the simplified source term for the numerical tests presented in Sect. 4, but more complicated boundary conditions are generally possible. Hence, for flat channels, where the gradients of the lower wall disappear, the source term only consists of momentum flux contributions in $z$-direction evaluated at the upper and lower wall, respectively.

The height-averaged scheme that solves the macro problem is entirely formulated in terms of the densities of conserved variables and does not contain any a priori assumption about the constitutive behavior of the lubricant which is encoded in the flux function through the equation of state $p(\rho)$ and the viscous stress tensor $\underline{\tau}(\mathbf{q})$. The functional form of the flux is obtained from the micro problem discussed in Sect. 2.3.

\subsection{Numerical Solution of the Macro Problem}

We use a finite-volume discretization of the two-dimensional domain with an explicit time integration scheme to solve Eq. (8). MacCormack's [10] method is easy to implement and including source terms into the predictor-corrector scheme is straightforward. The discretized version of the system reads

$$
\begin{aligned}
\boldsymbol{Q}_{i, j}^{*} & =\boldsymbol{Q}_{i, j}^{n}-\frac{\Delta t}{\Delta x}\left(\boldsymbol{F}_{x ; i+1, j}^{n}-\boldsymbol{F}_{x ; i, j}^{n}\right) \\
& -\frac{\Delta t}{\Delta y}\left(\boldsymbol{F}_{\boldsymbol{y} ; i, j+1}^{n}-\boldsymbol{F}_{\boldsymbol{y} ; i, j}^{n}\right)-\Delta t \boldsymbol{S}_{i, j}^{n},
\end{aligned}
$$

with 


$$
\begin{aligned}
\boldsymbol{Q}_{i, j}^{* *} & =\boldsymbol{Q}_{i, j}^{*}-\frac{\Delta t}{\Delta x}\left(\boldsymbol{F}_{\boldsymbol{x} ; i, j}^{*}-\boldsymbol{F}_{\boldsymbol{x} ; i-1, j}^{*}\right) \\
& -\frac{\Delta t}{\Delta y}\left(\boldsymbol{F}_{\boldsymbol{y} ; i, j}^{*}-\boldsymbol{F}_{\boldsymbol{y} ; i, j-1}^{*}\right)-\Delta t \boldsymbol{S}_{i, j}^{*},
\end{aligned}
$$

$$
\boldsymbol{Q}_{i, j}^{n+1}=\frac{1}{2}\left(\boldsymbol{Q}_{i, j}^{* *}+\boldsymbol{Q}_{i, j}^{n}\right),
$$

where $\boldsymbol{Q}_{i, j}^{*}$ and $\boldsymbol{Q}_{i, j}^{* *}$ are intermediate solutions obtained from forward and backward differencing of the discretized flux vectors $\boldsymbol{F}_{x / y ; i, j}^{* / n}$ and $\boldsymbol{S}_{i, j}^{* / n}$ is the discrete source term. Here, subscripts $i$ and $j$ denote the grid cell with side lengths $\Delta x$ and $\Delta y$ and the superscript $n$ denotes discrete time points separated by the time step $\Delta t$. The order of differencing directions is arbitrary and one could also switch between backward and forward differences after every consecutive time step. Although the intermediate steps are first order accurate, the overall scheme is second order accurate in space and in time.

Other Jacobian-free integration schemes such as Richtmyer's two-step version of the Lax-Wendroff scheme could also be implemented straightforwardly (see Ref. [11] for an overview of modifications to the Lax-Wendroff scheme with source terms). In the linear case (and without source term), MacCormack's method is equivalent to the Lax-Wendroff scheme [12]. The presented scheme can be modified to be total variation diminishing (TVD) as shown by Davis [13]. This can be of particular use in the presence of large gradients or at discontinuities, since spurious oscillations are damped by adding just the right amount of artificial viscosity. The TVD-correction term to the MacCormack corrector step is shown in appendix B.

Boundary conditions are implemented using a ghost cell approach, leading to $N+2$ cells in each direction, due to the three-point stencil of the MacCormack scheme. For Dirichlet-type boundary conditions, the value of the ghost cell is chosen such that the prescribed value of the conserved variable is satisfied at the cell boundary by linear interpolation between the boundary cell and the ghost cell. Inflow and outflow boundary conditions $\nabla \boldsymbol{q}=\mathbf{0}$ are satisfied by setting the ghost cell value to the boundary cell value. Implementation of periodic boundary conditions is also straightforward. One-dimensional examples in Sect. 4 are computed using the full two-dimensional description with periodic boundary conditions in $y$-direction, leading to a $(N+2) \times 3$-grid.

The scheme is implemented in Python using the numpy library [14]. Compared to conventional stationary Reynolds solvers, computation time can be large, since we are resolving the full transient behavior of the flow until steady state is reached, while the time step is limited by the Courant-Friedrichs-Lewy condition. Computational speed-up is achieved by spatial domain decomposition in combination with a parallel implementation using the mpi 4py bindings to the message passing interface (MPI) [15].

To benchmark the scaling behavior of our code, we calculated an incompressible flow problem until steady state for $400 \times 400$ and $1000 \times 1000$ finite-volume grid cells, respectively, on bwForCluster NEMO at the University of Freiburg ( $2 x$ Broadwell E5-2360v4 at $2.2 \mathrm{GHz}$ per compute node with a $100 \mathrm{Gbit} / \mathrm{s}$ OmniPath interconnect). On the smaller grid, the serial implementation reaches only 1.6 million lattice updates per second (MLUPS), which can be improved up to 54.4 MLUPS on 220 MPI processes (11 NEMO compute nodes). On the larger grid, our implementation peaks at 223.7 MLUPS when using 520 MPI processes (26 NEMO compute nodes). The number of time steps until steady state strongly depends on the nature of the problem, ranging from a few hundred steps to more than one million.

\subsection{Micro Problem}

The governing equation of the micro problem can be found by applying the splitting approach on Eq. (1)

$$
\frac{\partial \overline{\mathbf{q}}}{\partial t}=-\frac{\partial \overline{\mathbf{f}}_{x}}{\partial x}-\frac{\partial \overline{\mathbf{f}}_{y}}{\partial y}-\frac{\partial \mathbf{f}_{z}}{\partial \delta \mathbf{q}} \cdot \frac{\partial \delta \mathbf{q}}{\partial z} .
$$

By inserting Eq. (8), the governing equation of the macro problem, we obtain

$\frac{\partial \mathbf{f}_{z}}{\partial \delta \mathbf{q}} \cdot \frac{\partial \delta \mathbf{q}}{\partial z}=\mathbf{s}_{\mathbf{0}}=$ const.

where $\mathbf{s}_{\mathbf{0}}$ is the source term of the macro problem evaluated at the lateral position and time of the macro problem and the Jacobian reads

$$
\frac{\partial \mathbf{f}_{z}}{\partial \delta \mathbf{q}}=\left(\begin{array}{cccc}
0 & 0 & 0 & 1 \\
-\frac{j_{x} j_{z}}{\rho^{2}}+\frac{\partial \tau_{x z}}{\partial \rho} & \frac{j_{z}}{\rho}+\frac{\partial \tau_{x z}}{\partial j_{x}} & \frac{\partial \tau_{x z}}{\partial y_{y}} & \frac{j_{x}}{\rho}+\frac{\partial \tau_{x z}}{\partial j_{z}} \\
-\frac{j_{y} j_{z}}{\rho^{2}}+\frac{\partial \tau_{y z}}{\partial \rho} & \frac{\partial \tau_{y z}}{\partial j_{x}} & \frac{j_{z}}{\rho}+\frac{\partial \tau_{y z}}{\partial j_{y}} & \frac{j_{y}}{\rho}+\frac{\partial \tau_{y z}}{\partial j_{z}} \\
-\frac{j_{z}^{2}}{\rho^{2}}+\frac{\partial\left(\tau_{z z}+p\right)}{\partial \rho} & \frac{\partial \tau_{z z}}{\partial j_{x}} & \frac{\partial \tau_{z z}}{\partial j_{y}} & \frac{2 j_{z}}{\rho}+\frac{\partial \tau_{z z}}{\partial j_{z}}
\end{array}\right) .
$$

From the first row of Eq. (14), we find that $\partial_{z} \delta q_{4}=$ const., but since there can be no mass flux through the walls it vanishes everywhere, i.e., $q_{4}=j_{z}=0$. Thus, our splitting approach induces laminar flow, which is a reasonable assumption in thin-film flows and a reduced system is given by 
$\left(\begin{array}{ccc}\frac{\partial \tau_{x z}}{\partial \rho} & \frac{\partial \tau_{x z}}{\partial j_{x}} & \frac{\partial \tau_{x z}}{\partial j_{y}} \\ \frac{\partial \tau_{y z}}{\partial \rho} & \frac{\partial \tau_{y z}}{\partial j_{x}} & \frac{\partial \tau_{y z}}{\partial j_{y}} \\ \frac{\partial\left(\tau_{z z}+p\right)}{\partial \rho} & \frac{\partial \tau_{z z}}{\partial j_{x}} & \frac{\partial \tau_{z z}}{\partial j_{y}}\end{array}\right) \cdot\left(\begin{array}{c}\frac{\partial \delta \rho}{\partial z} \\ \frac{\partial \delta j_{x}}{\partial z} \\ \frac{\partial \delta j_{y}}{\partial z}\end{array}\right)=$ const.,

or simply

$\frac{\partial}{\partial z}\left(\begin{array}{c}\tau_{x z} \\ \tau_{y z} \\ p+\tau_{z z}\end{array}\right)=$ const.

The latter equation governs the local profiles of the remaining conserved variables and illustrates the relation between micro and macro problem. On one hand, local stresses are determined by the macroscopic flow conditions represented through the source term, whereas, on the other hand, these stresses govern the time evolution of the macroscopic variables. In a multiscale simulation, the micro problem could be replaced by a more accurate simulation method, such as molecular dynamics. However, we want to proceed using common assumptions about the viscous stress tensor that lead to the well-known results of continuum lubrication modeling.

\section{Constitutive Relations}

So far, we have only formally derived the structure of the micro problem in Sect. 2.3 without explicit use of constitutive laws. In the following section, we introduce common constitutive relations, both for the viscous stresses as well as the compressibility of the fluid. Thus, we are able to close the macro problem, and, with the numerical setup presented in Sect. 2.2, we address several aspects of lubrication modeling, including wall slip, non-Newtonian fluids, and cavitation. All material models shown here are implemented into the "Height-averaged Navier-Stokes" (HANS) solver which is publicly available under the terms of the MIT license ${ }^{1}$.

\subsection{Newtonian Fluids}

For Newtonian fluids, the viscous stress tensor is a linear function of the velocity gradient $\nabla \vec{u}$ given by

$\underline{\tau}=\eta\left(\nabla \vec{u}+(\nabla \vec{u})^{\top}\right)+\left(\zeta-\frac{2}{3} \eta\right)(\nabla \cdot \vec{u}) \underline{1}$,

where $\eta$ and $\zeta$ are the coefficients of shear and bulk viscosity, respectively. In order to express the components of the viscous stress tensor $\underline{\tau}$ in terms of $\overline{\boldsymbol{q}}$, we simply use our knowledge about $\tau$ in Eq. (17). From the first two rows, we find that

$\frac{\partial \tau_{x z}}{\partial z} \propto \frac{\partial^{2} u}{\partial z^{2}}=$ const.

$\frac{\partial \tau_{y z}}{\partial z} \propto \frac{\partial^{2} v}{\partial z^{2}}=$ const.

where $u(z)$ and $v(z)$ are the streamwise components of the local velocity field $\vec{u}\left(x_{0}, y_{0}, z\right)=(u, v, w)^{T}$, which can be described using second-degree polynomials,

$u(z)=\alpha_{1} z^{2}+\beta_{1} z+\gamma_{1}$,

$v(z)=\alpha_{2} z^{2}+\beta_{2} z+\gamma_{2}$

The cross-film velocity $w(z)$ vanishes as already shown in Sect. 2.3.

The parameters $\beta_{i}$ and $\gamma_{i}, i=1,2$ are determined from the boundary conditions at the bottom and top wall, namely

$u\left(h_{1}\right)=U_{1}+u_{\text {slip }, 1}$,

$u\left(h_{2}\right)=U_{2}+u_{\text {slip }, 2}$,

$v\left(h_{1}\right)=V_{1}+v_{\text {slip }, 1}$,

$v\left(h_{2}\right)=V_{2}+v_{\text {slip }, 2}$,

where $u_{\text {slip }, i}$ and $v_{\text {slip }, i}$ are slip velocities.

From the third row of Eq. (17) we obtain $\partial_{z} p=$ const.. If we neglect body forces in $z$-direction, the pressure at the top and bottom wall must be the same and therefore we find the typical result $p(z)=$ const., which is equivalent to a constant mass density across the channel height.

Thus, with the definition of the height-averaged mass flux

$\overline{\vec{j}}=\frac{\rho}{h} \int_{h_{1}}^{h_{2}} \vec{u} \mathrm{~d} z$,

we obtain the remaining parameters $\alpha_{i}$, which describe the Poiseuille contribution to the flow. This enables us to describe the viscous stress tensor according to Eq. (18) as a function of the average mass flux and density.

Hence, all entries of the flux matrix are given as a function of averaged conserved variables $\mathbb{F}=\mathbb{F}(\overline{\boldsymbol{q}})$. Computing the source term also requires unaveraged flux values at the top and bottom wall which are automatically defined by the choice of velocity and density profiles.

${ }^{1}$ https://github.com/hannes-holey/hans 


\subsection{Wall Slip}

Wall slip is typically quantified through the Navier slip length $b$ [16]. It becomes relevant for confined lubricants, when the gap height is in the order of the slip length, such as in EHL contacts or in the boundary lubrication regime. The concept of slip length arises from the assumption that the interfacial shear stress is proportional to the slip velocity with the constant of proportionality quantifying the friction between fluid and solid. Using the Newtonian fluid constitutive equation, the slip length can therefore be described as a combination of a purely bulk property (shear viscosity) with a purely interfacial one (friction) [17]. Geometrically, the slip length can be interpreted as the subsurface distance where the fluid velocity would equal that of the wall when linearly extrapolated, as shown in Fig. 1c. This leads to the following expression for the slip velocities

$u_{\text {slip }, i}= \pm b_{i}\left(\frac{\partial u}{\partial z}\right)_{z=h_{i}}$,

$v_{\text {slip }, i}= \pm b_{i}\left(\frac{\partial v}{\partial z}\right)_{z=h_{i}}$

where positive signs correspond to the upper and negative signs to the lower surface respectively. Naturally, many surfaces have heterogeneous surface chemistry, or surface properties can be tailored into particular stick-slip patterns, and therefore the slip length $b_{i}$ is a function of the lateral coordinates $x, y$.

\subsection{Non-Newtonian Fluids}

Complex lubricants often deviate from the Newtonian fluid behavior, especially when subjected to high pressures and/or high shear rates. Non-Newtonian effects can be modeled by a generalized Newtonian fluid description, where an effective viscosity $\eta_{\text {eff }}=\eta(\dot{\gamma}, p)$ replaces the constant one in the formulation of the Newtonian stress tensor, Eq. (18). For instance, shear-thinning or piezoviscous behavior is then described as a non-linear function of the shear rate $\dot{\gamma}$ and pressure $p$, respectively. Effects depending on the deformation history are not captured by the generalized Newtonian fluid model.

Accounting for piezoviscous effects is straightforward, since we have seen that pressure is constant across the gap height as a general result of our splitting approach. A typical model for the pressure dependence of viscosity is Barus equation [18]

$\eta(p)=\eta_{0} \exp (\alpha p)$ where $\alpha$ is the pressure viscosity coefficient, typically ranging between 10 and $20 \mathrm{GPa}^{-1}$. Although Eq. (24) is known to overestimate the viscosity for higher pressures and more accurate empirical modifications exist $[19,20]$, it is still often used.

Conversely, the strain varies across the gap height for general Couette-Poiseuille flow, which leads to velocity profiles that deviate from the Newtonian quadratic ones. Hence, an effective viscosity law depending on a height-averaged strain rate would not give the correct stress, especially at locations where pressure-driven flow dominates.

Here, we employ a power-law fluid model to show that arbitrary constitutive laws can be incorporated into the micro problem. As the name suggests, the shear stress components depend on the shear rate through a power law

$\tau=\phi \dot{\gamma}^{n}$.

where $n$ is the flow index, describing shear-thinning $(n<0)$ or shear-thickening $(n>0)$ behavior. $\phi$ is called the flow consistency index and has units of $\operatorname{Pas}^{n}$. For $n=1$, we recover the Newtonian fluid.

Note that the power law does not correctly describe the fluid behavior in the limiting cases of zero or infinite shear rate. Typically, the effective viscosity converges to finite values $\left(\eta_{0}, \eta_{\infty}\right)$ in these limits, which is for instance accounted for in the Carreau [21] or Eyring [22, 23] shear-thinning models. The type of shear-thinning model may also depend on pressure and/or temperature. In a recent molecular dynamics study on model fluids under EHL conditions, Jadhao and Robbins [24] showed that there is a generic transition from power-law behavior at low pressure, and therefore low viscosity, to Eyring behavior at high pressure.

Nevertheless, we use the simple power-law model here, since it can be treated analytically, but emphasize that principally more realistic solutions to the micro problem could be found. Similar to the Newtonian case in Sect. 3.1, we integrate Eq. (17) of the micro problem to obtain the velocity profiles. Davaa et al. [25] showed that for plane Couette-Poiseuille flow of power-law fluids, two cases have to be distinguished:

1. The velocity profile has a maximum within the gap that is larger than the sliding velocity, i.e., $\max u(z)>U$ for $z \in[0, h]$,

2. The velocity profile either has a maximum within the gap that is lower than the sliding velocity, i.e., $\max u(z)<U$, or it does not have a maximum within the gap at all.

Case 1 requires a piecewise integration from the lower wall $z=0$ to the position of the maximum velocity $z=h_{1}^{*}$ and from the location of the maximum velocity $z=h_{1}^{*}$ to the upper wall $z=h$, whereas the velocity profile of case 2 can 
be written as a single expression that likewise depends on the location of the velocity maximum $h_{2}^{*}$, which not necessarily lies within the gap. The velocity profiles have the form

$$
\begin{aligned}
& u^{(1)}(z)= \begin{cases}-\frac{n}{n+1} \alpha_{1}^{\frac{1}{n}}\left(h_{1}^{*}-z\right)^{\frac{n+1}{n}}+\beta_{1}, & 0 \leq z \leq h_{1}^{*} \\
-\frac{n}{n+1} \alpha_{1}^{\frac{1}{n}}\left(z-h_{1}^{*}\right)^{\frac{n+1}{n}}+\gamma_{1}, & h_{1}^{*}<z \leq h\end{cases} \\
& u^{(2)}(z)=-\frac{n}{n+1} \alpha_{2}^{\frac{1}{n}}\left(h_{2}^{*}-z\right)^{\frac{n+1}{n}}+\beta_{2} .
\end{aligned}
$$

A more detailed description of the integration procedure for the velocity profiles can be found in Appendix $\mathrm{C}$ or in Ref. [25]. The parameters $\beta_{1}, \gamma_{1}$, and $\beta_{2}$ are obtained in a similar fashion as in the Newtonian case using boundary conditions of the form of Eq. (21a)-(21d). Finally, $\alpha_{1}$ and $\alpha_{2}$ are obtained from the definition of the height-averaged momentum flux, Eq. (22). However, in both cases, the unknown parameters $h_{1}^{*}$ and $h_{2}^{*}$ are left to be determined. In case 1 we use the continuity condition of the velocity profile at $z=h_{1}^{*}$ as an additional expression for $\alpha_{1}$ and in case 2 we use the remaining boundary condition at the top wall to obtain $\alpha_{2}$. By equating these two expressions for $\alpha_{i}$ in both cases, respectively, we obtain two non-linear equations that determine $h_{1}^{*}$ and $h_{2}^{*}$

$$
\begin{aligned}
& {\left[\frac{(1+n) U}{\left(h-h_{1}^{*}\right)^{\frac{n+1}{n}}-h_{1}^{* \frac{n+1}{n}}}\right]^{n}=\left[\frac{(1+2 n)\left(\bar{u} h-U h_{1}^{*}\right)}{h_{1}^{* \frac{2 n+1}{n}}+\left(h-h_{1}^{*}\right)^{\frac{2 n+1}{n}}}\right]^{n},} \\
& U=\frac{\bar{u} h\left[h_{2}^{* \frac{n+1}{n}}-\left(h_{2}^{*}-h\right)^{\frac{n+1}{n}}\right]}{\frac{n}{2 n+1}\left[h_{2}^{* \frac{2 n+1}{n}}-\left(h_{2}^{*}-h\right)^{\frac{2 n+1}{n}}\right]-h\left(h_{2}^{*}-h\right)^{\frac{n+1}{n}}},
\end{aligned}
$$

where $\bar{u}$ denotes the height-averaged velocity. Setting $h^{*}=0$ in either of the equations leads to the critical condition $U / \bar{u}=(1+2 n) /(1+n)$ that marks the boundary between the two cases. The resulting velocity profiles, such as the ones shown in Fig. 2, are then used to compute the viscous stress tensor components for the power-law fluid.

\subsection{Equation of State and Cavitation}

Next to the viscous stress tensor another constitutive relation is naturally contained in the flux function. For compressible fluids, the system of continuity equation and momentum balance needs to be closed by an equation of state, relating the pressure in the hyperbolic flux contribution to the mass density. Various types of equations describing the compressibility of the fluid can be used, ranging from the ideal gas to semi-empirical descriptions, such as the isothermal Dowson-Higginson [26, 27] equation of state

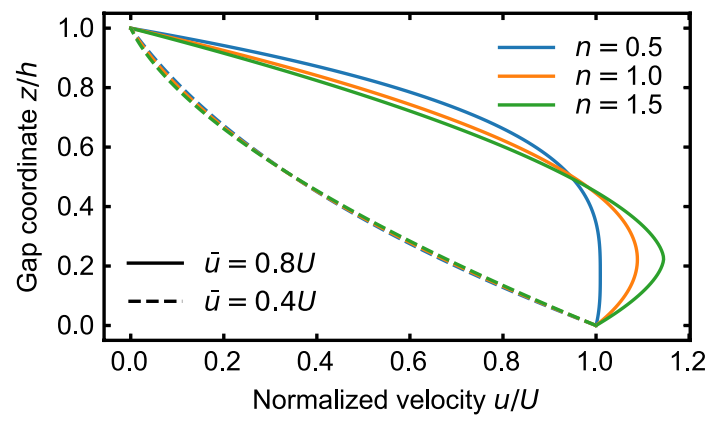

Fig. 2 Example of velocity profiles for a power-law fluid under Couette-Poiseuille flow for three different flow indices (shearthinning: $n=0.5$, Newtonian: $n=1$, shear-thickening: $n=1.5$ ). Solid lines represent case $1(\bar{u} / U>(1+n) /(1+2 n))$ with significant Poiseuille contribution and dashed lines represent case 2 $(\bar{u} / U<(1+n) /(1+2 n))$, where the flow is Couette dominated.

$p(\rho)=p_{0}+C_{1} \frac{\rho-\rho_{0}}{C_{2} \rho_{0}-\rho}$,

with fitting parameters $C_{1}$ and $C_{2}$, which is commonly used for mineral base oils.

However, such expressions do not reflect the effect of vaporous cavitation, which mainly occurs in diverging contact geometries, where the pressure may fall below the vapor pressure of the liquid. The first model that accounted for mass-conserving cavitation was based on the work of Jakobsson, Floberg, and Olsson (JFO) [28-31], who formulated boundary conditions for the rupture and reformation of a fluid film under the assumption that the pressure is constant in the cavitated regions. Elrod's and Adam's cavitation algorithm [32,33] builds upon the JFO approach, but added a switch function to the Reynolds equation that suppresses the Poiseuille contribution to the flow in the cavitated regions. The advantage of the Elrod-Adams (EA) approach is that a single equation can be used for the whole domain.

Here, we incorporate mass-conserving cavitation directly through the equation of state, by either fixing the pressure to a constant value $p_{\text {cav }}$ at densities lower than the saturation density, which is conceptually similar to the EA algorithm or using a unique equation of state describing the behavior of vapor, liquid, and vapor-liquid mixture as in the model of Bayada and Chupin [34].

Their model assumes constant compressibility both in the liquid and the vapor phases, defined by the velocities of sound $c_{1}$ and $c_{\mathrm{v}}$, respectively. The assumption that both phases of the vapor-liquid mixture have the same velocity allows to homogenize the dynamics of the flow in the cavitated region such that the same set of equations as in the full-film region can be used. The pressure-density relation 


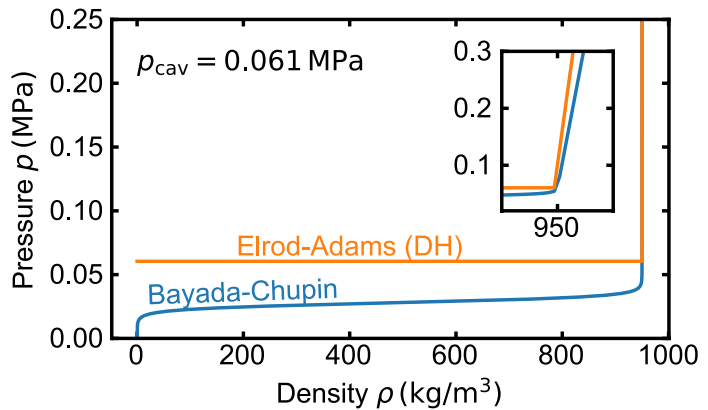

Fig. 3 Equation of state (EOS) used for modeling mass-conserving cavitation in combination with the height-averaged Navier-Stokes solver. Bayada and Chupin [34] use a smooth function to interpolate between the pure vapor and liquid phases, whereas the Elrod-Adams algorithm with Dowson-Higginson EOS (cf. Ref [36]) leads to a sharp edge at the cavitation pressure.

for the mixture of vapor bubbles and liquid is defined in terms of the vapor fraction $\alpha=\left(\rho-\rho_{1}\right) /\left(\rho_{\mathrm{v}}-\rho_{1}\right)$, in which $\rho_{\mathrm{v}}$ and $\rho_{1}$ are the density of the vapor and the liquid at the wet point and the bubble point, respectively. Bayada and Chupin used a relation proposed by Van Wijngaarden [35]

$\frac{1}{c_{\mathrm{f}}^{2}}=\rho\left(\frac{\alpha}{c_{\mathrm{v}}^{2} \rho_{\mathrm{v}}}+\frac{1-\alpha}{c_{1}^{2} \rho_{1}}\right)$

relating the fluid's velocity of sound $c_{\mathrm{f}}$ with the vapor fraction. Integrating equation $\mathrm{d} p / \mathrm{d} \rho=c_{\mathrm{f}}^{2}$ in each of the regions and requiring continuity of the pressure at the transition from vapor to mixture $(\alpha=1)$ as well as from mixture to liquid $(\alpha=0)$ and that $p(0)=0$, one finds

$p(\rho)= \begin{cases}c_{\mathrm{v}}^{2} \rho, & \alpha \geq 1, \\ p_{\mathrm{cav}}+\left(\rho-\rho_{1}\right) c_{1}^{2}, & \alpha \leq 0, \\ p_{\mathrm{vm}}+N \ln \left(\frac{\rho_{\mathrm{v}} c_{\mathrm{v}}^{2} \rho}{\rho_{1}\left(\rho_{\mathrm{v}} c_{\mathrm{v}}^{2}(1-\alpha)+\rho_{1} c_{1}^{2} \alpha\right)}\right), & 0<\alpha<1,\end{cases}$

with pressure at the transition points from vapor to mixture

$p_{\mathrm{vm}}=\rho_{\mathrm{v}} c_{\mathrm{v}}^{2}$,

and from mixture to liquid (i.e., the cavitation pressure)

$$
\begin{gathered}
p_{\text {cav }}=\rho_{\mathrm{v}} c_{\mathrm{v}}^{2}-N \ln \left(\frac{\rho_{\mathrm{v}}^{2} c_{\mathrm{v}}^{2}}{\rho_{1}^{2} c_{1}^{2}}\right), \text { with } \\
N=\frac{\rho_{\mathrm{v}} c_{\mathrm{v}}^{2} \rho_{1} c_{1}^{2}\left(\rho_{\mathrm{v}}-\rho_{1}\right)}{\rho_{\mathrm{v}}^{2} c_{\mathrm{v}}^{2}-\rho_{1}^{2} c_{1}^{2}} .
\end{gathered}
$$

Note that, for convenience, in Eq. (30) the definition of $\alpha$ is extended to values larger than one or smaller than zero. Obviously, the interpretation as the vapor fraction is then no longer valid in these regimes. Fig. 3 shows an example of Eq. (30) for material parameters that lead to a cavitation pressure of 0.061 MPa. For comparison, an EOS following the Dowson-Higginson relation in the liquid phase and having constant pressure everywhere else is given, which represents a realization of the EA algorithm through the EOS.

The pressure-density relation of Bayada and Chupin [34] is accompanied by a viscosity model that interpolates between the vapor and liquid viscosity, the simplest one being a linear interpolation in terms of the vapor fraction $\alpha$

$\eta(\alpha)=\eta_{\mathrm{v}} \alpha+(1-\alpha) \eta_{1}, \quad 0 \leq \alpha \leq 1$.

\section{Numerical Tests}

In this section we demonstrate the validity and versatility of the HANS solver. We use the local solutions to the micro problem with the constitutive relations presented in Sect. 3 to solve the macro problem with the numerical framework presented in Sect. 2.2. When available, we compare the converged steady-state pressure and density profiles to literature results.

\subsection{Inclined Slider}

As a first test for the transient numerical scheme, we use an inclined slider geometry with an ideal gas EOS and compare the steady-state solution with the results of Ref. [8] obtained from a compressible Reynolds equation. The height profile is given by $h(x)=h_{\max }-s x$ for $x \in[0, L]$, where $s$ is the slope of the pad. Here, we test the scheme at different sliding speeds $U$ for a bearing with $L=0.1 \mathrm{~m}, h_{\max }=66 \mu \mathrm{m}$, and $s=5.6 \cdot 10^{-4}$. The equation of state is given as

$p(\rho)=\frac{p_{0}}{\rho_{0}} \rho$,

with ambient pressure $p_{0}=101325 \mathrm{~Pa}$ and ambient density $\rho_{0}=1.1853 \mathrm{~kg} / \mathrm{m}^{3}$. The shear viscosity is assumed to be constant at value of $\eta=18.46 \cdot 10^{-6}$ Pas and the bulk viscosity $\zeta$ is set to zero. The obtained pressure profiles for three different velocities of the gas-lubricated slider bearing are shown in Fig. 4a. A substantial increase of load-bearing capacity with sliding speed can be observed and the results match perfectly with the ones obtained in Ref. [8].

The simulation results for the inclined slider geometry, as well as all other tests in the remainder of this section, are obtained under the assumption that convective (non-linear) inertial terms in the momentum equations can be neglected, which is a reasonable assumption in most cases. However, as shown in Ref. [8] for slider bearings and the ideal gas EOS, these necessary additional assumptions can lead to significant errors at high sliding speeds. Since the convective acceleration term is generally included into the definition of 
(a)
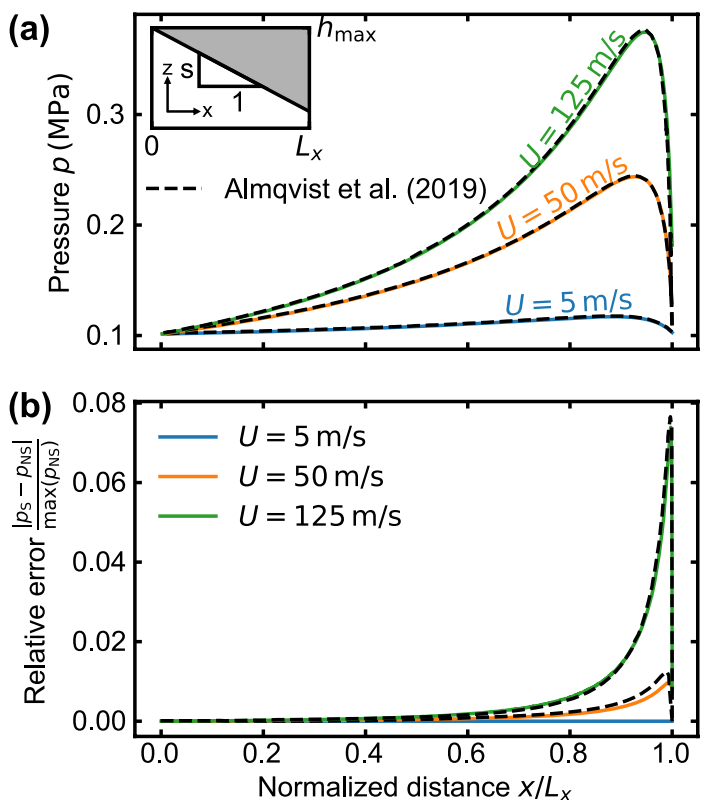

Fig. 4 Pressure profiles for a gas-lubricated inclined slider geometry for three different sliding speeds and comparison to results from Ref. [8] (a). The influence of including convective acceleration terms is studied in (b) for increasing sliding speed. In both cases, the fluid is described by an isothermal ideal gas equation of state and has constant viscosity. Pressure profiles for the highest velocity $U=125 \mathrm{~m} / \mathrm{s}$ are obtained using the total variation diminishing (TVD) version of the MacCormack scheme

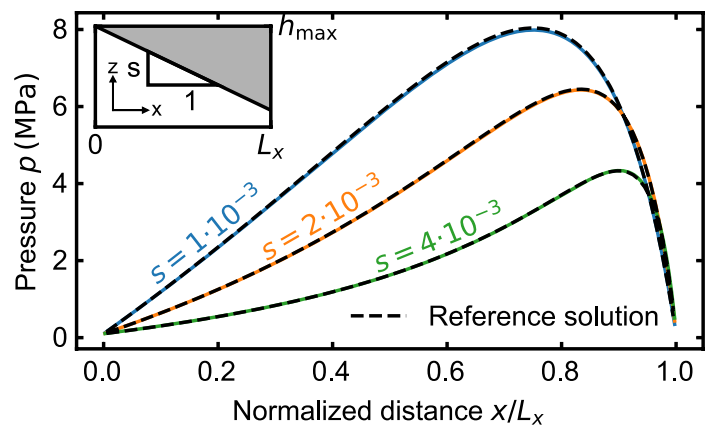

Fig. 5 Pressure profiles for an inclined slider geometry with three different slopes at sliding speed $U=0.1 \mathrm{~m} / \mathrm{s}$ and Dowson-Higginson equation of state. Reference solutions are obtained from a finitedifference solver for the compressible Reynolds equation used in Ref. [37]

the flux function in Eq. (2), we have tested its influence for the inclined slider geometry for three different velocities. In Fig. $4 \mathrm{~b}$, the relative difference between the height-averaged Stokes solution (without non-linear term, $p_{\mathrm{s}}$ ) and the heightaveraged Navier-Stokes solution $\left(p_{\mathrm{NS}}\right)$ is shown. The maximum pressure error due to neglection of inertial terms for a sliding speed of $U=125 \mathrm{~m} / \mathrm{s}$ goes up to $8 \%$ and agrees with the error reported in Ref.[8]. We want to highlight that both

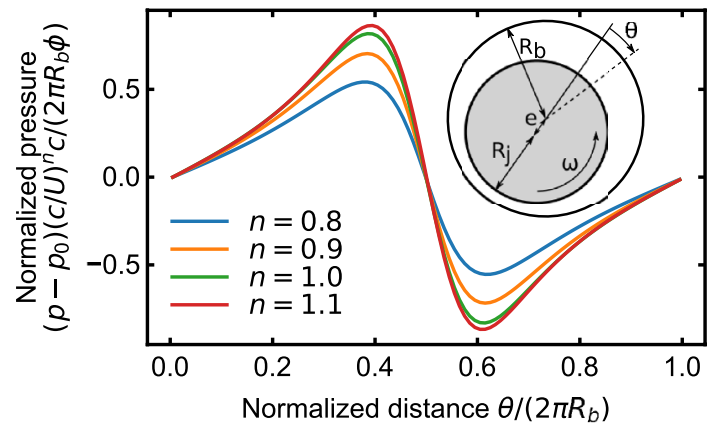

Fig. 6 Non-dimensional pressure profiles for four power-law fluids in an infinitely long journal bearing. The flow index determines the strength of the shear-thinning $(n<1)$ or shear-thickening $(n>1)$ effect. For $n=1$ we recover the Newtonian case

linear and non-linear solutions are obtained using the same numerical framework.

We further test the scheme for fluids with varying compressibility using the Dowson-Higginson EOS (Eq. (28)). Here, we choose the parameters reported in Ref. [36] $\left(C_{1}=2.22 \mathrm{GPa}, C_{2}=1.66\right)$ and a slider geometry with inlet gap height $h_{\max }=1.5 \mu \mathrm{m}$, sliding speed $U=0.1 \mathrm{~m} / \mathrm{s}$, and three different slopes. The obtained pressure profiles are shown in Fig. 5 and are compared with results obtained from the compressible Reynolds solver reported in Codrignani et al. [37]. As in the previous case, the obtained pressure profiles match the Reynolds reference solution.

\subsection{Journal Bearing with Power-Law Fluid}

We have introduced the power-law fluid in Sect. 3.3 as an example for a generalized Newtonian fluid. The availability of analytical expressions for the velocity profiles allows us to implement the relevant stress components as a result of the micro problem for power-law fluids. This highlights the advantage of our splitting approach, since the numerical solution of the macro problem remains untouched.

We test our implementation of power-law stresses for an infinitely long journal bearing geometry which can be described by a sinusoidal height profile

$h(\theta)=c+e \cos \left(\theta / R_{b}\right)$,

with radial clearance $c=R_{b}-R_{j}$ and eccentricity $e . R_{b}$ and $R_{j}$ are the radii of the bearing and journal, respectively. The eccentricity ratio is defined as $\varepsilon=e / c$.

The resulting pressure profiles for four different flow indices are shown in Fig. 6. All simulations were performed for a journal bearing with eccentricity ratio $\varepsilon=0.6$, bearing radius $R_{b}=1 / 2 \pi \mathrm{mm}$, clearance $c=0.01 R_{b}$, and at a sliding speed of $U=5 \mathrm{~m} / \mathrm{s}$. The flow consistency index 
Table 1 Parameters for the equation of state and the viscosity of liquid and vapor phase following Ref. [34]

\begin{tabular}{llllll}
\hline $\begin{array}{l}\eta_{\mathrm{l}} \\
(\text { Pas })\end{array}$ & $\begin{array}{l}\eta_{\mathrm{v}} \\
(\mathrm{Pas})\end{array}$ & $\begin{array}{l}c_{\mathrm{l}} \\
(\mathrm{m} / \mathrm{s})\end{array}$ & $\begin{array}{l}c_{\mathrm{v}} \\
(\mathrm{m} / \mathrm{s})\end{array}$ & $\begin{array}{l}\rho_{\mathrm{l}} \\
\left(\mathrm{kg} / \mathrm{m}^{3}\right)\end{array}$ & $\begin{array}{l}\rho_{\mathrm{v}} \\
\left(\mathrm{kg} / \mathrm{m}^{3}\right)\end{array}$ \\
\hline 0.039 & $3.9 \cdot 10^{-5}$ & 1600 & 352 & 850 & 0.019 \\
\hline
\end{tabular}

$\phi=0.0794 \mathrm{Pas}^{n}$ is the same in all four cases and can be interpreted as the Newtonian shear viscosity for $n=1$. For a better comparison we show the dimensionless pressure profiles $\tilde{p}=\left(p-p_{0}\right)(c / U)^{n} c /\left(2 \pi R_{b} \phi\right)$. The effect of non-Newtonian lubricants with shear-thinning $(n<1)$ or shear-thickening $(n>1)$ effect is directly visible from the normalized pressure profiles. However, this example cannot be used to describe an increase or decrease of load-bearing capacity due to the non-Newtonian behavior of the lubricant, since cavitation is not considered in the diverging part of the geometry. The implementation of cavitation models is validated in the following examples.

\section{3 (Twin-) Prabolic Slider}

To study the effect of cavitation in hydrodynamic lubrication, we use a converging-diverging height profile, where we expect cavitation bubble formation in the diverging part of the bearing. Therefore, we use a parabolic slider geometry, which has been widely used to benchmark cavitation models. The height profile of the one-dimensional bearing is given by

$h(x)=\frac{4\left(h_{\max }-h_{\min }\right)}{L_{x}^{2}}\left(x-\frac{L_{x}}{2}\right)^{2}+h_{\min }$,

with maximum gap height $h_{\max }=50.8 \mu \mathrm{m}$, minimum gap height $h_{\min }=25.4 \mu \mathrm{m}$, and length $L_{x}=76.2 \mathrm{~mm}$. The parameters describing the lubricant through Eq. (30) are given in Tab. 1. The resulting pressure profiles for two different discretizations $(N=100$ and $N=200)$ are shown in Fig. 7a and the relative density or saturation $\rho / \rho_{l}$ is shown in Fig. 7b. Both agree very well with the profiles presented in Ref. [38].

The cavitation pressure $p_{\text {cav }}$, or, in the notation of Bayada and Chupin, $p_{\mathrm{ml}}$, for the presented results is approximately $0.061 \mathrm{MPa}$. As shown in their paper, the obtained results can be directly compared to solutions using the JFO/EA formalism, when using the same cavitation pressure and a constant compressibility EOS in the full-film region.

Here, we proceed with another numerical test using an EOS with varying compressibility in combination with the EA algorithm, as for instance presented in Ref. [36]. The so-called twin-parabolic slider is used, consisting of
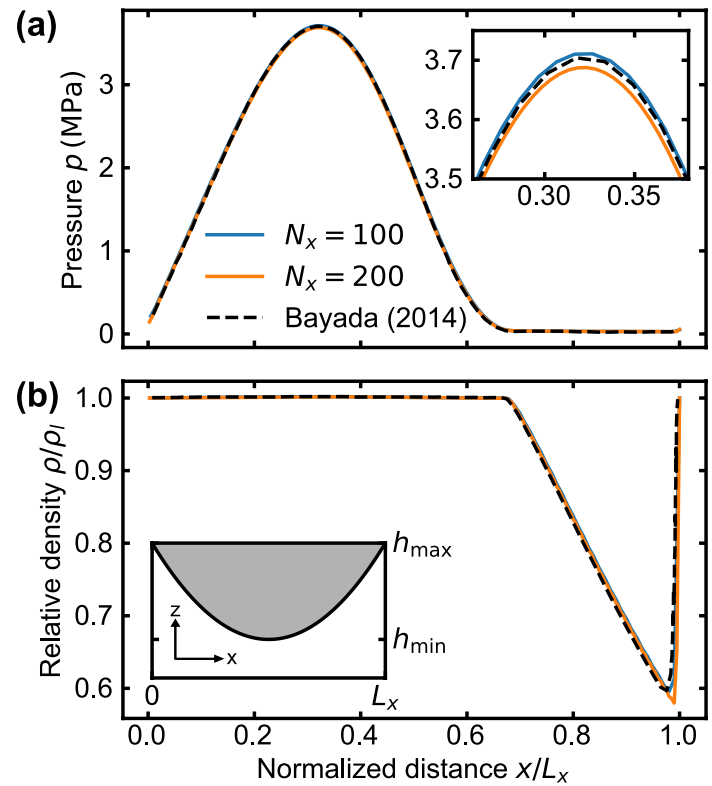

Fig. 7 Pressure profile (a) and saturation profile (b) for the parabolic slider geometry and comparison to the results of Ref. [38]

two neighboring "bumps" with a parabolic shape and the same minimum and maximum gap heights as in the previous example. The length and sliding speed of the bearing are also the same as before. The pressure boundary conditions at the inlet and outlet are $p_{\text {in }}=3.36 p_{0}$ and $p_{\text {out }}=p_{\text {cav }}$, respectively, with ambient pressure $p_{0}=10^{5} \mathrm{~Pa}$ and cavitation pressure $p_{\text {cav }}=0 \mathrm{~Pa}$. The pressure profile is shown in Fig. 8 which agrees well with the result of Ref. [36].

\subsection{Flat Channel with Roughness}

Surface roughness plays an important role in lubrication, since small-scale height variations can lead to non-zero load-bearing capacities even when the contacting surfaces are macroscopically flat, which was first observed experimentally by Hamilton et al. [39]. Hence, we benchmark the presented numerical scheme on geometries that mimic surface roughness. Again, we use an example presented in Ref. [36], with a height profile given by

$h(x)= \begin{cases}h_{0}+a \sin ^{2}\left(\frac{4 \pi n}{L}\left(\frac{L}{2}-x\right)\right), & 0 \leq x<L / 2, \\ h_{0}, & L / 2 \leq x \leq L .\end{cases}$

We study two realizations of this profile, with amplitude parameter $a=h_{0} / 2$ and two different frequencies. The number of periods $n$ affects the gap height at the inlet, where $n=5$ leads to a diverging inlet and $n=4.75$ to a converging inlet, as shown in Fig. 9a. We perform simulations for gap height $h_{0}=10 \mu \mathrm{m}$, bearing length $L=0.1 \mathrm{~m}$, sliding speed 


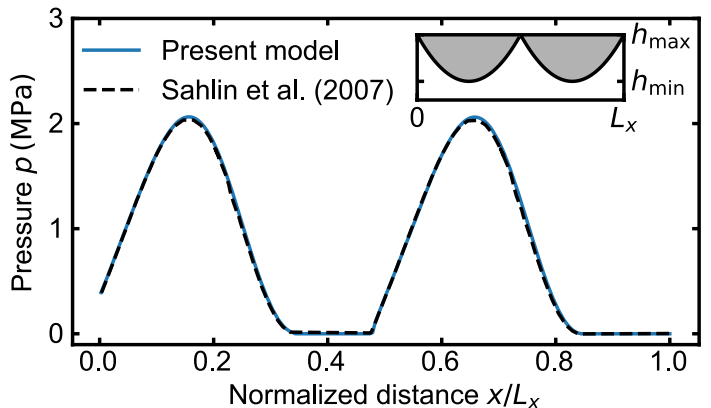

Fig. 8 Pressure profile for the twin-parabolic slider geometry and comparison to the results of Ref. [36]

(a)

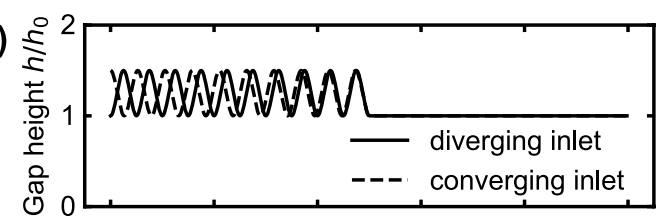

(b)

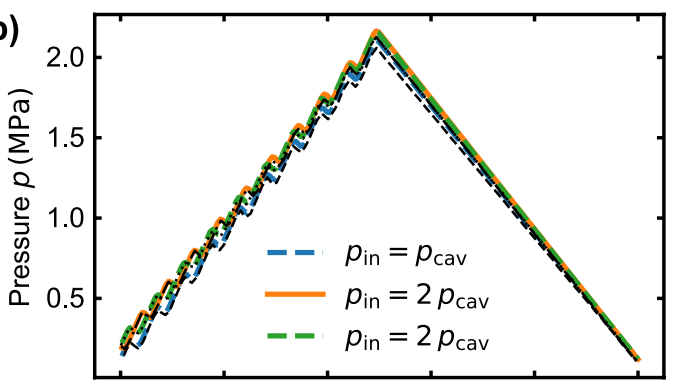

(c)

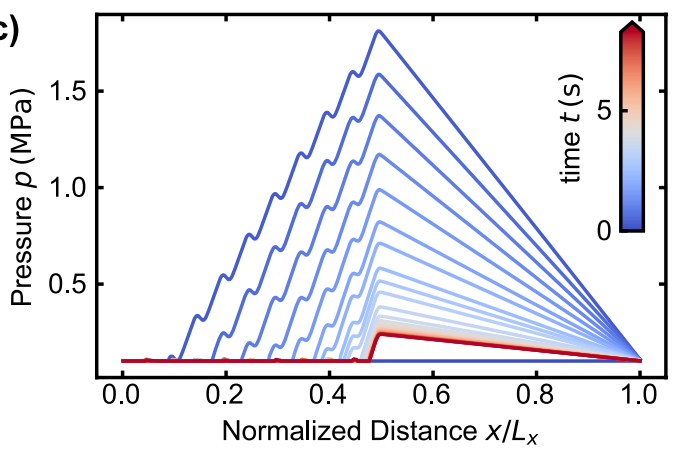

Fig. 9 Geometry of the flat channel with $\sin ^{2}$-roughness (a), corresponding pressure profiles for starved converging inlet (dashed blue line), pressurized diverging inlet (solid orange line), and pressurized converging inlet (dashed green line) (b). Black lines in (b) correspond to the results from Ref. [36] and appear as dashed, dash-dotted, and dotted lines, respectively (in the same order). The time evolution of the pressure profile for a starved diverging inlet is shown in (c)

$U=0.25 \mathrm{~m} / \mathrm{s}$, and the fluid's viscosity is $\eta=0.04$ Pas. The resulting pressure profiles do not only depend on the gap height and geometry of the inlet, but also on the pressure boundary condition.
In this example, we use both a pressurized inlet $\left(p_{\text {in }}=2 p_{\text {cav }}\right)$ and a starved inlet $\left(p_{\text {in }}=p_{\text {cav }}\right)$ with $p_{\text {cav }}=10^{5} \mathrm{~Pa}$. The former case leads to a pressure profile with load-bearing capacity for both type of geometries as can be seen in Fig 9b. Also, if the inlet geometry is converging and the ambient pressure is the cavitation pressure, a load-bearing capacity can be achieved, since incoming fluid is immediately compressed and further surface corrugations do not lead to cavitation. The obtained pressure profiles are similar for all three cases and agree very well with the results of Ref. [36].

The last case, a starved inlet with diverging geometry, is different from the previous ones as it does not generate a loadbearing pressure profile. However, the results of the transient simulation reveal that initially a substantial pressure build-up is produced, which is similar to the other three situations. With increasing time, a small cavitation zone grows from the inlet into the domain and the pressure peak decreases. Furthermore, as shown in the time evolution of the pressure profile in Fig. 9c, the velocity with which the cavitation zone expands decreases until it almost comes to a standstill at $t=8 \mathrm{~s}$, with a remaining non-zero load-bearing capacity. This example highlights an advantage of the explicit solution algorithm in comparison to the mostly implicit solutions of the stationary Reynolds equation, since transient phenomena in lubrication are automatically resolved.

\subsection{Flat Channel with Heterogeneous Wall Slip}

Surface slip reduces friction and the possibility to control the surface chemistry [40] opens a wide range of applications in lubrication, e.g., by tailoring the confining walls of a flat channel into slipping and sticking domains $[41,42]$. Here, we study a flat channel of length $L=2 \lambda$ and height $h$ with heterogeneous wetting properties at the top surface as reported in Ref. [43]. The lower surface sticks and is sheared at constant velocity $U_{1}$. For better comparison, we here present the results for the pressure in dimensionless form, i.e., $\tilde{p}=p / p_{0}$, with $p_{0}=\eta U_{1} \lambda / h^{2}$. The sticking and slipping domains have equal length $\lambda$, and periodic boundary conditions are applied in the $x$ - and $y$-direction (infinitely long bearing).

The obtained pressure profiles for three different sliding velocities are compared to a Reynolds model including wall slip for an incompressible fluid in Fig. 10a. Since our method is generally designed for compressible fluids, we arbitrarily choose an extremely stiff EOS to mimic incompressibility. Here, we use again the Dowson-Higginson EOS and choose parameters ensuring that deviations from the reference density are negligibly small. The Reynolds model predicts a pressure gradient 

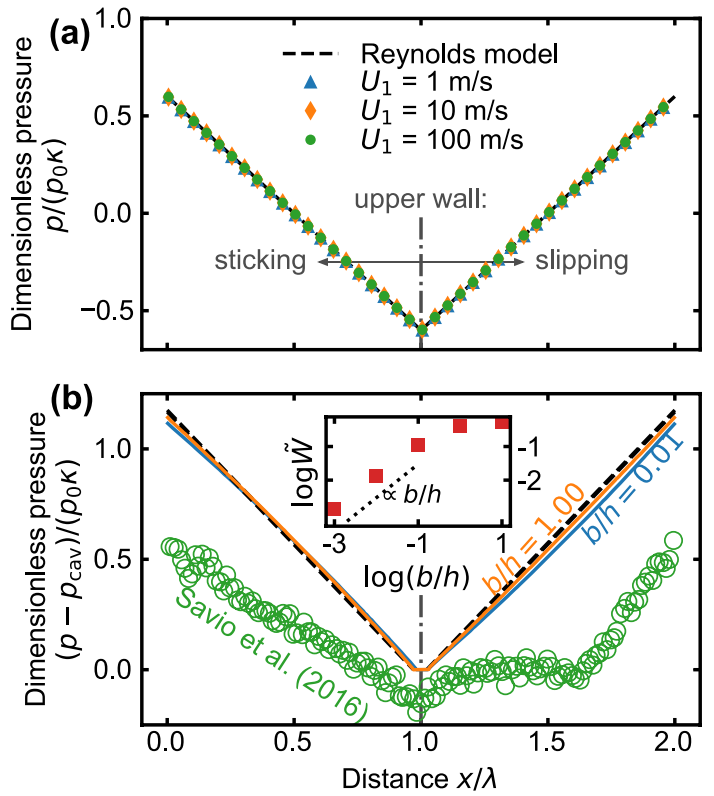

Fig. 10 Non-dimensional pressure distributions for a flat channel with heterogeneous wall slip and comparison to a Reynolds model, including slip. A nearly incompressible equation of state (EOS) is used in (a), which allows the pressure to go below zero. The pressure profiles for the n-decane system from Ref. [43] obtained from Molecular Dynamics (MD) simulations, as well as continuum results for two different slip lengths are shown in (b), where the van der Waals equation with a cutoff at $P_{\text {cav }}=1 \mathrm{MPa}$ is employed to model cavitation. The inset in (b) shows the non-dimensional load-bearing capacity per unit width for various slip lengths

$\frac{\partial p}{\partial x}= \begin{cases}-\frac{6 \eta U_{1}}{h^{2}}\left(\frac{b \lambda_{2}}{\lambda_{1}(h+4 b)+\lambda_{2}(h+b)}\right), & 0 \leq x \leq \lambda_{1} \\ \frac{6 \eta U_{1}}{h^{2}}\left(\frac{b \lambda_{1}}{\lambda_{1}(h+4 b)+\lambda_{2}(h+b)}\right), & L-\lambda_{2} \leq x \leq L,\end{cases}$

with $\lambda_{1}$ and $\lambda_{2}$ being the size of the sticking and slipping domain, respectively. Thus, for $\lambda_{1}=\lambda_{2}=L / 2$, the nondimensional pressure gradient reads

$\frac{\partial \tilde{p}}{\partial \tilde{x}}= \begin{cases}-6 \kappa / 5, & 0 \leq \tilde{x} \leq 1, \\ 6 \kappa / 5, & 1 \leq \tilde{x} \leq 2,\end{cases}$

with $\tilde{x}=x / \lambda$ and $\kappa=5 b /(2 h+5 b)$. The dimensionless pressure profiles normalized by the wall slip quantifying parameter $\kappa$ agree perfectly with the prediction of Eq. (37). However, both the analytical model and the numerical results predict unphysical negative pressures, and a non-zero load-bearing capacity in symmetric systems with periodic boundary conditions can only be achieved by considering cavitation.

Therefore, we proceed with parameters that represent the n-decane system studied with molecular dynamics (MD) simulations in Ref. [43]. The channel has length
$L=143.9 \mathrm{~nm}$, height $h=5.5 \mathrm{~nm}$, and sliding velocity $U_{1}=10 \mathrm{~m} / \mathrm{s}$. We use the van der Waals equation as EOS

$p(\rho)=\frac{R T \rho}{M-b \rho}-\frac{a \rho^{2}}{M^{2}}$,

with $a=5.273 \mathrm{~Pa} \mathrm{~m}^{6} / \mathrm{mol}^{2}, \quad b=3.04 \cdot 10^{-4} \mathrm{~m}^{3} / \mathrm{mol}$, $M=142.29 \mathrm{~g} / \mathrm{mol}, T=303 \mathrm{~K}$, and the shear viscosity is $\eta=0.3 \mathrm{mPas}$. To avoid negative pressure and the unphysical increase of pressure with volume in the subcritical van der Waals loop, we use the aforementioned EA approach with a cutoff at $P_{\text {cav }}=1 \mathrm{MPa}$, which agrees with the external pressure applied in the MD simulations. Note, that in case of the van der Waals equation, the EA approach is similar to the well-known Maxwell construction [44], except that our choice of saturation pressure is not based on the equal area rule.

The dimensionless pressure profiles for the n-decane system are shown in Fig. 10b for two different slip lengths. For both systems a cavitation zone forms directly at the transition from stick to slip behavior at the top wall and the size of the cavitation zone decreases with increasing slip length. We extract the size of the full-film regions $\lambda_{1}$ and $\lambda_{2}$ from our simulation to compute the Reynolds solution according to Eq. (38). The analytical and numerical pressure profiles agree reasonably well. The slight curvature of the numerical pressure profiles can be accounted to the compressibility of the van der Waals equation in contrast to the incompressible analytical result.

The inset of Fig. 10b shows the non-dimensional loadbearing capacity per unit width

$\tilde{W}=\frac{W}{p_{0} L}=\frac{1}{p_{0} L} \int_{0}^{L} p(x) \mathrm{d} x$

for various slip lengths. The simulation results suggest that with increasing stick-slip contrast the system's ability to support an external load increases linearly for small slip lengths $b<<h$ until it reaches a stable value as $b$ is in the order of the gap height or larger, which agrees with the analytical Reynolds model.

The MD simulations of Savio et al. [43] predict a substantially larger cavitation zone and, consequently, lower pressure excursion in the remaining full-film region. The pressure falls below the external pressure in front of the stick-slip boundary, where it reaches its minimum before it goes to zero in the cavitated zone. Such complex behavior governed by the nucleation, lifetime, and collapse of vapor bubbles cannot be addressed with simple macroscopic cavitation models.

This example foreshadows the potential application of the presented solver in multiscale frameworks, such as the combined use of MD simulations and continuum methods. Molecular interactions govern effects, such as the nucleation 
of cavitation bubbles or local variations of surface slip, that are important to accurately describe lubrication phenomena on experimental length and time scales.

\section{Conclusion}

In this paper, a novel method to solve lubrication problems has been derived. Splitting the hydrodynamic variables into a time-dependent height-averaged and a local cross-film part implies laminar flow, which is a reasonable assumption in lubrication, but avoids further assumptions on constitutive behavior. We derived height-averaged balance equations for mass and momentum which contain an additional source term as a result of the reduction of dimensionality. Analytical solutions for the micro problem have been derived for the Newtonian fluid and the power-law fluid as an example for a generalized Newtonian (time-independent non-Newtonian) fluid.

The time-explicit finite-volume scheme to solve the macro problem has been tested for common constitutive laws and it has been shown that various results from established solution schemes can be reproduced. The numerical tests included lubrication problems for compressible fluids with and without consideration of inertial effects, mass-conserving cavitation, wall slip, as well as non-Newtonian fluids. The explicit solution algorithm allows to study dynamic problems, such as the time evolution of lubricant flow between geometrically and chemically structured surfaces.

The additional complexity of our method in comparison to standard Reynolds solvers is legitimated by its flexibility due to the clear separation of local constitutive behavior and macroscopic flow evolution. Hence, it should not be seen as a competitor against Reynolds-based schemes, but rather as a complementary method in situations where the constitutive behavior does not allow a straightforward application of a Reynolds-type equation.

Furthermore, we believe that solving the micro problem is not limited to the analytical examples shown here. Finding approximate or closed-form solutions to the micro problem allows testing of new constitutive models within the presented numerical framework. Concurrent multiscale simulations may represent another useful application, where the micro problem could be outsourced and solved using more accurate simulation methods, such as molecular dynamics.

\section{A. Detailed Derivation of the Height-Averaged Balance Equations}

Starting from Eq. (4) and applying Leibniz integral rule for differentiation under the integral sign on the l.h.s., we obtain $\frac{1}{h} \int_{h_{1}}^{h_{2}} \partial_{t} \boldsymbol{q} z=\frac{1}{h}\left(\partial_{t} \int_{h_{1}}^{h_{2}} \boldsymbol{q} \mathrm{d} z-\left.\boldsymbol{q}\right|_{z=h_{2}} \frac{\mathrm{d} h_{2}}{\mathrm{~d} t}+\left.\boldsymbol{q}\right|_{z=h_{1}} \frac{\mathrm{d} h_{1}}{\mathrm{~d} t}\right)$.

The first term on the r.h.s. of Eq. (A1) can be rewritten using the product rule

$\frac{1}{h} \partial_{t} \int_{h_{1}}^{h_{2}} \boldsymbol{q} \mathrm{d} z=\partial_{t}\left(\frac{1}{h} \int_{h_{1}}^{h_{2}} \mathbf{q} \mathrm{d} z\right)+\frac{1}{h^{2}} \frac{\mathrm{d} h}{\mathrm{~d} t} \int_{h_{1}}^{h_{2}} \boldsymbol{q} \mathrm{d} z$.

Using $\frac{\mathrm{d} h}{\mathrm{~d} t}=\frac{\mathrm{d} h_{2}}{\mathrm{~d} t}-\frac{\mathrm{d} h_{1}}{\mathrm{~d} t}$ and plugging the result back into Eq. (A1) leads to

$$
\begin{aligned}
\frac{1}{h} \int_{h_{1}}^{h_{2}} \partial_{t} \boldsymbol{q} \mathrm{d} z & =\partial_{t}\left(\frac{1}{h} \int_{h_{1}}^{h_{2}} \boldsymbol{q} \mathrm{d} z\right)+\frac{1}{h}\left(\frac{1}{h} \int_{h_{1}}^{h_{2}} \boldsymbol{q} \mathrm{d} z-\left.\boldsymbol{q}\right|_{z=h_{2}}\right) \frac{\mathrm{d} h_{2}}{\mathrm{~d} t} \\
& -\frac{1}{h}\left(\frac{1}{h} \int_{h_{1}}^{h_{2}} \boldsymbol{q} \mathrm{d} z-\left.\boldsymbol{q}\right|_{z=h_{1}}\right) \frac{\mathrm{d} h_{1}}{\mathrm{~d} t} .
\end{aligned}
$$

Multiplying Eq. (A3) by $h$ and using the shorthand notation for height averages $\bar{\phi}=\frac{1}{h} \int_{h_{1}}^{h_{2}} \phi \mathrm{d} z$ yields exactly Eq. 6 . The derivation for the terms on the r.h.s. of Eq. (4) follows equivalently.

\section{B. TVD-MacCormack Correction}

To obtain a TVD-variant of the MacCormack scheme, we add a correction term

$$
\begin{aligned}
\Delta \mathbf{Q}_{i, j}^{\mathrm{TVD}} & =\left[K\left(r_{i}^{+}\right)+K\left(r_{i+1}^{-}\right)\right] \Delta \mathbf{Q}_{i+1 / 2, j}^{n} \\
& -\left[K\left(r_{i-1}^{+}\right)+K\left(r_{i}^{-}\right)\right] \Delta \mathbf{Q}_{i-1 / 2, j}^{n} \\
& +\left[K\left(r_{j}^{+}\right)+K\left(r_{j+1}^{-}\right)\right] \Delta \mathbf{Q}_{i, j+1 / 2}^{n} \\
& -\left[K\left(r_{j-1}^{+}\right)+K\left(r_{j}^{-}\right)\right] \Delta \mathbf{Q}_{i, j-1 / 2}^{n}
\end{aligned}
$$

to the corrector step (Eq. (12a)) with

$$
\begin{array}{r}
r_{i}^{ \pm}=\frac{\Delta \mathbf{Q}_{i-1 / 2, j}^{n} \cdot \Delta \mathbf{Q}_{i+1 / 2, j}^{n}}{\Delta \mathbf{Q}_{i \pm 1 / 2, j}^{n} \cdot \Delta \mathbf{Q}_{i \pm 1 / 2, j}^{n},} \\
r_{j}^{ \pm}=\frac{\Delta \mathbf{Q}_{i, j-1 / 2}^{n} \cdot \Delta \mathbf{Q}_{i, j+1 / 2}^{n}}{\Delta \mathbf{Q}_{i, j \pm 1 / 2}^{n} \cdot \Delta \mathbf{Q}_{i, j \pm 1 / 2}^{n}}, \\
\Delta \mathbf{Q}_{i \pm 1 / 2, j}^{n}= \pm\left(\mathbf{Q}_{i \pm 1, j}^{n}-\mathbf{Q}_{i, j}^{n}\right), \\
\Delta \mathbf{Q}_{i, j \pm 1 / 2}^{n}= \pm\left(\mathbf{Q}_{i, j \pm 1}^{n}-\mathbf{Q}_{i, j}^{n}\right),
\end{array}
$$

and

$$
K(r)=\frac{C}{2}(1-\phi(r))
$$


where $\phi(r)=\max (0, \min (2 r, 1))$ is the flux limiter function and the parameter $C$ depends on the local Courant number $v$ through

$C= \begin{cases}v(1-v), & v \leq 0.5 \\ 0.25, & v>0.5 .\end{cases}$

For more details see Ref. [13].

\section{Integration of Velocity Profiles for Power-Law Fluids}

The constitutive relation for a power-law fluid Eq. (25) together with the governing equation of the micro problem Eq. (17) leads to a non-linear ordinary differential equation for the velocity profile. For the lower part of case 1 we have

$\frac{\mathrm{d}^{2} u}{\mathrm{~d} z^{2}}=-\frac{\alpha_{1}}{n}\left(\frac{\mathrm{d} u}{\mathrm{~d} z}\right)^{1-n}, \quad 0 \leq z \leq h_{1}^{*}$,

where $\alpha_{1}$ is a constant. We choose an ansatz for the strain rate of the form

$\frac{\mathrm{d} u}{\mathrm{~d} z}=A\left(h_{1}^{*}-z\right)^{\chi}$,

which leads to

$A \chi\left(h_{1}^{*}-z\right)^{\chi-1}=\frac{\alpha_{1}}{n} A^{1-n}\left(h_{1}^{*}-z\right)^{\chi(1-n)}$.

Hence, we find $\chi=1 / n$ and $A=\alpha_{1}^{1 / n}$ such that the velocity profile is given by

$u(z)=-\frac{n}{n+1} \alpha_{1}^{\frac{1}{n}}\left(h_{1}^{*}-z\right)^{\frac{n+1}{n}}+\beta_{1}, \quad 0 \leq z \leq h_{1}^{*}$.

The integration constant $\beta_{1}$ is determined using the boundary condition at the lower wall $u(0)=U$.

Furthermore, the velocity profile for the upper part of case 1 can be found in a similar manner using

$\frac{\mathrm{d}^{2} u}{\mathrm{~d} z^{2}}=-\frac{\alpha_{1}}{n}\left(-\frac{\mathrm{d} u}{\mathrm{~d} z}\right)^{1-n}, \quad 0 \leq z \leq h_{1}^{*}$,

and an ansatz

$\frac{\mathrm{d} u}{\mathrm{~d} z}=A\left(z-h_{1}^{*}\right)^{\chi}$.

The resulting velocity profile reads

$u(z)=-\frac{n}{n+1} \alpha_{1}^{\frac{1}{n}}\left(z-h_{1}^{*}\right)^{\frac{n+1}{n}}+\gamma_{1}, \quad h_{1}^{*} \leq z \leq h$.
The derivation of the velocity profile for case 2 is identical to that of the lower part of case 1.

Acknowledgements The authors gratefully acknowledge support by the German Research Foundation (DFG) through GRK 2450 and the European Research Council (ERC) through Starting Grant 757343. Calculations were carried out on bwForCluster NEMO at the University of Freiburg (DFG grant INST 39/963-1 FUGG).

Funding Open Access funding enabled and organized by Projekt DEAL.

Data Availability The data to reproduce the figures are available from the authors upon reasonable request.

Code Availability All simulations were performed with HANS (https:// github.com/hannes-holey/hans).

\section{Declarations}

Conflict of interest The authors declare no competing interests.

Open Access This article is licensed under a Creative Commons Attribution 4.0 International License, which permits use, sharing, adaptation, distribution and reproduction in any medium or format, as long as you give appropriate credit to the original author(s) and the source, provide a link to the Creative Commons licence, and indicate if changes were made. The images or other third party material in this article are included in the article's Creative Commons licence, unless indicated otherwise in a credit line to the material. If material is not included in the article's Creative Commons licence and your intended use is not permitted by statutory regulation or exceeds the permitted use, you will need to obtain permission directly from the copyright holder. To view a copy of this licence, visit http://creativecommons.org/licenses/by/4.0/.

\section{References}

1. Reynolds, O.: IV. On the theory of lubrication and its application to Mr. Beauchamp Tower's experiments, including an experimental determination of the viscosity of olive oil. Philo. Trans. R. Soc. Lond. 177, 157-234 (1886). https://doi.org/10.1098/rstl. 1886.0005

2. Lugt, P.M., Morales-Espejel, G.E.: A review of elasto-hydrodynamic lubrication theory. Tribol. Trans. 54(3), 470-496 (2011). https://doi.org/10.1080/10402004.2010.551804

3. Gropper, D., Wang, L., Harvey, T.J.: Hydrodynamic lubrication of textured surfaces: A review of modeling techniques and key findings. Tribol. Int. 94, 509-529 (2016). https://doi.org/10.1016/j. triboint.2015.10.009

4. Hamrock, B.J., Schmid, S.R., Jacobson, B.O.: Fundamentals of Fluid Film Lubrication. CRC Press, Boca Raton. (2004). https:// doi.org/10.1201/9780203021187

5. Rajagopal, K.R., Szeri, A.Z.: On an inconsistency in the derivation of the equations of elastohydrodynamic lubrication. Proc. R. Soc. Lond. Series A 459(2039), 2771-2786 (2003). https://doi. org/10.1098/rspa.2003.1145

6. Bayada, G., Cid, B., García, G., Vázquez, C.: A new more consistent Reynolds model for piezoviscous hydrodynamic lubrication problems in line contact devices. Appl. Math. Model. 37(18), 8505-8517 (2013). https://doi.org/10.1016/j.apm.2013.03.072 
7. Gustafsson, T., Rajagopal, K.R., Stenberg, R., Videman, J.: Nonlinear Reynolds equation for hydrodynamic lubrication. Appl. Math. Model. 39(17), 5299-5309 (2015). https://doi.org/10. 1016/j.apm.2015.03.028

8. Almqvist, A., Burtseva, E., Pérez-Ráfols, F., Wall, P.: New insights on lubrication theory for compressible fluids. Int. J. Eng. Sci. 145, 103170 (2019). https://doi.org/10.1016/j.ijengsci.2019. 103170

9. Dowson, D.: A generalized Reynolds equation for fluid-film lubrication. Int. J. Mech. Sci. 4(2), 159-170 (1962). https://doi.org/10. 1016/S0020-7403(62)80038-1

10. MacCormack, R.W.: The effect of viscosity in hypervelocity impact cratering. J. Spacecraft Rockets 40(5), 757-763 (2003). https://doi.org/10.2514/2.6901

11. Zhang, Y., Tabarrok, B.: Modifications to the Lax-Wendroff scheme for hyperbolic systems with source terms. Int. J. Numer. Methods Eng. 44(1), 27-40 (1999). https://doi.org/10.1002/(SICI) 1097-0207(19990110)44:13c27::AID-NME4853e3.0.CO;2-0

12. LeVeque, R.J.: Finite Volume Methods for Hyperbolic Problems, Cambridge Texts in Applied Mathematics. Cambridge University Press, Cambridge (2002). https://doi.org/10.1017/CBO9780511 791253

13. Davis, S.F.: TVD finite difference schemes and artificial viscosity. National Aeronautics and Space Administration. ICASE Rep. 84-20 (1984)

14. Harris, C.R., Millman, K.J., van der Walt, S.J., Gommers, R., Virtanen, P., Cournapeau, D., Wieser, E., Taylor, J., Berg, S., Smith, N.J., Kern, R., Picus, M., Hoyer, S., van Kerkwijk, M.H., Brett, M., Haldane, A., del Río, J.F., Wiebe, M., Peterson, P., Gérard-Marchant, P., Sheppard, K., Reddy, T., Weckesser, W., Abbasi, H., Gohlke, C., Oliphant, T.E.: Array programming with NumPy. Nature 585(7825), 357-362 (2020). https://doi.org/10. 1038/s41586-020-2649-2

15. Dalcín, L., Paz, R., Storti, M.: MPI for Python. J. Parallel Distrib. Comput. 65(9), 1108-1115 (2005). https://doi.org/10.1016/j.jpdc. 2005.03.010

16. Navier, C.: Mémoire sur les lois du mouvement des fluides. Mém. l'Acad. R. Sci. l'Inst. France 6(1823), 389-440 (1823)

17. Bocquet, L., Barrat, J.-L.: Flow boundary conditions from nanoto micro-scales. Soft Matter 3(6), 685-693 (2007). https://doi.org/ 10.1039/B616490K

18. Barus, C.: Isothermals, isopiestics and isometrics relative to viscosity. Am. J. Sci. 45(266), 87-96 (1893). https://doi.org/10.2475/ ajs.s3-45.266.87

19. Roelands, C.J.A.: Correlational aspects of the viscosity-temperature-pressure relationship of lubricating oils. PhD Thesis, Technical University of Delft (1966)

20. Doolittle, A.K.: Studies in Newtonian flow. II. The dependence of the viscosity of liquids on free-space 22(12), 1471-1475 (1951). https://doi.org/10.1063/1.1699894

21. Carreau, P.J.: Rheological equations from molecular network theories. Trans. Soc. Rheol. 16(1), 99-127 (1972). https://doi.org/10. $1122 / 1.549276$

22. Eyring, H.: Viscosity, plasticity, and diffusion as examples of absolute reaction rates. J. Chem. Phys. 4(4), 283-291 (1936). https://doi.org/10.1063/1.1749836

23. Ewell, R.H., Eyring, H.: Theory of the viscosity of liquids as a function of temperature and pressure. J. Chem. Phys. 5(9), 726736 (1937). https://doi.org/10.1063/1.1750108

24. Jadhao, V., Robbins, M.O.: Rheological properties of liquids under conditions of elastohydrodynamic lubrication. Tribol. Lett. 67(3), 66 (2019). https://doi.org/10.1007/s11249-019-1178-3
25. Davaa, G., Shigechi, T., Momoki, S.: Plane Coutte-Poiseuille flow of power-law non-Newtonian fluids. Reports of the Faculty of Engineering, Nagasaki University 30(54) (2000)

26. Dowson, D., Higginson, G., Whitaker, A.: Elasto-hydrodynamic lubrication: a survey of isothermal solutions. J. Mech. Eng. Sci. 4(2), 121-126 (1962)

27. Dowson, D., Higginson, G.R.: Elasto-Hydrodynamic Lubrication: The Fundamentals of Roller and Gear Lubrication. Pergamon Press, Oxford (1966)

28. Jakobsson, B., Floberg, L.: The finite journal bearing, considering vaporization. Trans. Chalm. Univ. Technol. 190, 1-116 (1957)

29. Floberg, L.: The two-groove journal bearing, considering cavitation. Technical Report 231, Institute of Machine Elements, Chalmers University of Technology, Gothenburg, Sweden (1960)

30. Floberg, L.: Lubrication of two cylinder surfaces considering cavitation. Technical Report 232, Institute of Machine Elements, Chalmers University of Technology, Gothenburg, Sweden (1961)

31. Olsson, K.-O.: Cavitation in dynamically loaded bearings. Trans. Chalm. Univ. Technol. 308, 1 (1965)

32. Elrod, H., Adams, M.: A computer program for cavitation and starvation problems, cavitation and related phenomena in lubrication. In: Dowson, D., Godet, M., Taylor, C.M. (eds.) Lubrication, pp. 37-43. Mechanical Engineering Publications, London (1975)

33. Elrod, H.G.: A cavitation algorithm. J. Lubr. Technol. 103, 350 354 (1981)

34. Bayada, G., Chupin, L.: Compressible fluid model for hydrodynamic lubrication cavitation. J. Tribol. 135, 4 (2013). https://doi. org/10.1115/1.4024298

35. Van Wijngaarden, L.: One-dimensional flow of liquids containing small gas bubbles. Ann. Rev. Fluid Mech. 4(1), 369-396 (1972)

36. Sahlin, F., Almqvist, A., Larsson, R., Glavatskih, S.: A cavitation algorithm for arbitrary lubricant compressibility. Tribol. Int. 40(8), 1294-1300 (2007). https://doi.org/10.1016/j.triboint.2007. 02.009

37. Codrignani, A., Savio, D., Magagnato, F., Frohnapfel, B.: A scaling parameter for pressure losses and thermal effects in lubricant flows with viscous dissipation. Tribol. Int. 113, 238-244 (2017). https://doi.org/10.1016/j.triboint.2016.12.007

38. Bayada, G.: From a compressible fluid model to new mass conserving cavitation algorithms. Tribol. Int. 71, 38-49 (2014). https://doi.org/10.1016/j.triboint.2013.10.014

39. Hamilton, D.B., Walowit, J.A., Allen, C.M.: A theory of lubrication by microirregularities. J. Basic Eng. 88(1), 177-185 (1966). https://doi.org/10.1115/1.3645799

40. Xia, Y., Whitesides, G.M.: Soft lithography. Ann. Rev. Mater. Sci. 28, 153-184 (1998). https://doi.org/10.1146/annurev.matsci.28.1.153

41. Belyaev, A.V., Vinogradova, O.I.: Effective slip in pressure-driven flow past super-hydrophobic stripes. J. Fluid Mech. 652, 489-499 (2010). https://doi.org/10.1017/S0022112010000741

42. Cieplak, M., Koplik, J., Banavar, J.R.: Nanoscale fluid flows in the vicinity of patterned surfaces. Phys. Rev. Lett. 96(11), 114502 (2006). https://doi.org/10.1103/PhysRevLett.96.114502

43. Savio, D., Pastewka, L., Gumbsch, P.: Boundary lubrication of heterogeneous surfaces and the onset of cavitation in frictional contacts. Sci. Adv. 2(3), 1501585 (2016). https://doi.org/10.1126/ sciadv. 1501585

44. Clerk-Maxwell, J.: On the dynamical evidence of the molecular constitution of bodies. Nature 11(279), 357-359 (1875). https:// doi.org/10.1038/011357a0

Publisher's Note Springer Nature remains neutral with regard to jurisdictional claims in published maps and institutional affiliations. 